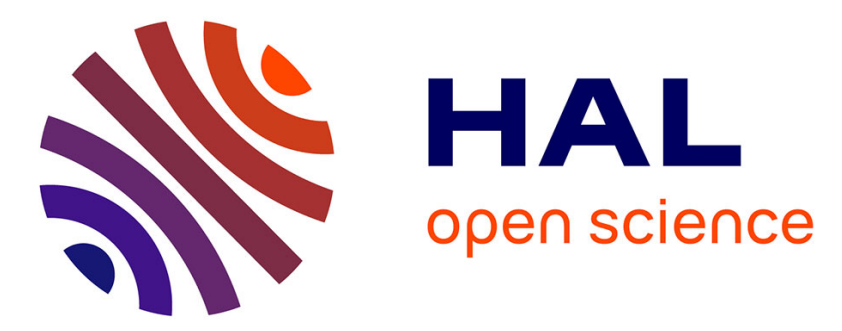

\title{
Demonstration of the Existence of Dumbbell Silicene: A Stable Two-Dimensional Allotrope of Silicon
}

\author{
Thomas Leoni, Conor Hogan, Kai Zhang, Michel Daher Mansour, Romain \\ Bernard, Romain Parret, Andrea Resta, Stefano Colonna, Yves Borensztein, \\ Fabio Ronci, et al.
}

\section{To cite this version:}

Thomas Leoni, Conor Hogan, Kai Zhang, Michel Daher Mansour, Romain Bernard, et al.. Demonstration of the Existence of Dumbbell Silicene: A Stable Two-Dimensional Allotrope of Silicon. Journal of Physical Chemistry C, 2021, 125 (32), pp.17906-17917. 10.1021/acs.jpcc.1c02088 . hal-03333968

\section{HAL Id: hal-03333968 https://hal.science/hal-03333968}

Submitted on 4 Oct 2021

HAL is a multi-disciplinary open access archive for the deposit and dissemination of scientific research documents, whether they are published or not. The documents may come from teaching and research institutions in France or abroad, or from public or private research centers.
L'archive ouverte pluridisciplinaire HAL, est destinée au dépôt et à la diffusion de documents scientifiques de niveau recherche, publiés ou non, émanant des établissements d'enseignement et de recherche français ou étrangers, des laboratoires publics ou privés. 


\section{Demonstration of the Existence of Dumbbell Silicene: a Stable Two-Dimensional Allotrope of Silicon}

Thomas Leoni ${ }^{\S}$, Conor Hogan ${ }^{\dagger^{*}}$, Kai Zhang ${ }^{\ddagger}$, Michel Daher Mansour ${ }^{\S \perp}$, Romain Bernard ${ }^{\ddagger}$, Romain Parret $^{\S}$, Andrea Resta ${ }^{T}$, Stefano Colonnat, Yves Borensztein ${ }^{\dagger}$, Fabio Roncit, Geoffroy Prévot ${ }^{t^{*}}$, Laurence Masson ${ }^{{ }^{*}}$

§Aix Marseille Univ, CNRS, CINaM, Campus de Luminy, Case 913, 13288 Marseille Cedex 9, France

$\dagger$ Istituto di Struttura della Materia, Consiglio Nazionale delle Ricerche (ISM-CNR), Via del Fosso del Cavaliere 100, 00133 Roma, Italy

\$Sorbonne Université, CNRS, Institut des NanoSciences de Paris, INSP, F-75005, Paris, France

`Synchrotron SOLEIL, L’Orme des Merisiers Saint-Aubin, BP 4891192 Gif-sur-Yvette Cedex, France 


\section{ABSTRACT}

We report a combined experimental and theoretical study on the formation of dumbbell silicene structures on $\operatorname{Ag}(110)$. High-resolution scanning tunneling microscopy (STM) reveals a rich tapestry of adatom-free and -decorated bidimensional silicene phases covering the whole $\operatorname{Ag}(110)$ surface. The most thermodynamically stable silicene models obtained from densityfunctional theory (DFT) perfectly reproduce all features observed by STM. These phases correspond to different Si buckled honeycomb reconstructions $((13 \times 4), c(18 \times 4)$ and $c(8 \times 4))$ composed of two periodic motifs common to all structural models. DFT calculations show that these reconstructions are stabilized by the presence of ordered arrays of Si adatoms adsorbed on top of silicene in a dumbbell configuration. Grazing incidence X-ray diffraction (GIXD) measurements confirm the growth of a dumbbell silicene layer. The structure factor values are well reproduced by a $(13 \times 4)$ model with $4 \mathrm{Si}$ adatoms per unit cell and a slight distortion of the hexagonal unit cell. Our STM-DFT-GIXD study demonstrates the formation of dumbbell silicene, a theoretically predicted two-dimensional $\mathrm{Si}$ allotrope. This opens up perspectives for tuning the peculiar properties of silicene. 


\section{INTRODUCTION}

Since the graphene breakthrough, two-dimensional (2D) materials have become a major field in materials research, largely motivated by their outstanding electronic and optoelectronic properties that foresee their integration in a wide range of next-generation devices. ${ }^{1} 2 \mathrm{D}$ materials beyond graphene made of elements of Group 14 such as silicon and germanium have experienced a renewed interest in the last decade due to the similarity between their valence electronic configuration and that of graphene as well as the widespread use of Si and Ge in the semiconductor industry. Indeed, theoretical studies have predicted metastable configurations of free-standing silicene and germanene, the $\mathrm{Si}$ - and Ge- based counterpart of graphene, consisting of 2D honeycomb low buckled structures associated with the presence of a Dirac cone in their band structure. $^{2}$ The considerable interest in these Group 14 2D materials, from a theoretical and experimental point of view, has been reinforced by the prediction of a much stronger spin-orbit coupling than in graphene, favoring more prominent quantum spin Hall effects and by the possibility of energy band gap engineering. ${ }^{3,4}$ These peculiar electronic properties are related to the presence of a mixed $\mathrm{sp}^{2}-\mathrm{sp}^{3}$ hybridization, which results in a low-buckled $2 \mathrm{D}$ structure, contrary to the $\mathrm{sp}^{2}$ electronic configuration and flat geometry of graphene. ${ }^{5}$ As widely reported, a key issue for these materials lies in their synthesis. As silicene and germanene cannot be obtained by exfoliation of the bulk material, a substrate that generally dictates the structure of the monolayer is necessarily required for the epitaxial growth of $\mathrm{Si}$ and Ge 2D sheets.

Although synthesis of silicene has been attempted on several different substrates, including $\mathrm{ZrB}_{2}(0001),{ }^{6} \mathrm{Ir}(111),{ }^{7} \mathrm{ZrC}(111),{ }^{8} \mathrm{MoS}_{2},{ }^{9} \mathrm{Ru}(0001),{ }^{10} \mathrm{Au}(111){ }^{11}$ and very recently $\mathrm{NaCl} / \mathrm{Ag}(110)$, 12 silver surfaces have by far achieved the most success. Indeed, the synthesis of silicene has been reported on $\operatorname{Ag}(111)$ in 2012, ${ }^{13,14,15,16,17}$ together with the observation in angle-resolved 
photoelectron spectroscopy (ARPES) spectra of electronic dispersion similar to the one of graphene. ${ }^{13}$ Although it was later shown that the linear features revealed in ARPES measurements were actually due to the interaction of the Si overlayer with the silver substrate rather than to the presence of Dirac cone electronic structure, ${ }^{18,19,20,21}$ these pioneering works have boosted the experimental and theoretical investigations of silicene on silver substrates in the years to follow. ${ }^{22}$

The possibility to tune the electronic properties of graphene and especially to open a band gap has been intensively studied and demonstrated through the formation of graphene nanoribbons $(\mathrm{NRs})^{23,24}$ or doping of the 2D layer.. ${ }^{25,26}$ In contrast, very few experimental studies have demonstrated similar trends for silicene, in spite of the wide number of theoretical studies. In particular, although silicene NRs were widely believed to form on $\operatorname{Ag}(110),{ }^{27,28,29}$ the observed NR reconstruction was later shown to be composed of pentamer chains of Si lying in the missing rows (MRs) of the substrate. ${ }^{30}$ As a result, the surface electronic properties are far from those expected for lateral confinement of a silicene sheet. ${ }^{30,31}$ An alternative way to tune the silicene properties is via the formation of dumbbell (DB) like structures. These configurations are built from the original 2D honeycomb structure by adding adatoms at selected lattice sites. DB structures have been theoretically predicted for honeycomb 2D layers of silicon, germanium, tin

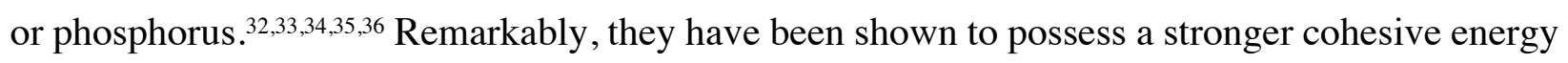
than their pristine counterparts. In particular, DB silicene has recently been predicted to constitute the true ground state of $2 \mathrm{D}$ silicon. ${ }^{33}$ Interestingly, the predicted electronic properties of DB silicene differ from those of free-standing silicene with a gap opening, a spin-polarized ground state and a very low thermal conductivity. ${ }^{32,33,37}$ Although dumbbell-like units have been proposed to form on an exotic 2D Si allotrope with a Kagome-like lattice different from the honeycomb structure expected for silicene, ${ }^{38}$ an experimental confirmation of the formation of DB silicene is 
still lacking. Indeed, it must be noted that although DB silicene has been proposed to explain the $\sqrt{3} \times \sqrt{3}$ phase of $\operatorname{Si} / \operatorname{Ag}(111),{ }^{39}$ a recent scanning tunneling microscopy (STM) and densityfunctional theory (DFT) study demonstrated that the observed phase corresponds to thicker films. ${ }^{40}$

In this article, we evidence the formation of DB silicene. We show that by adding $\mathrm{Si}$ atoms to the Si pentamer chain superstructure grown on $\operatorname{Ag}(110)$, this phase transforms into a silicene single-layer which covers the whole Ag surface and is stabilized by regular DB-like atomic units. Through a combination of STM, DFT and grazing incidence X-ray diffraction (GIXD), we demonstrate that the silicene overlayer is composed of honeycomb structures exhibiting different buckling patterns stabilized by the presence of the adatoms. Our findings give a comprehensive demonstration of the existence of DB silicene structures.

\section{METHODS}

STM. The STM measurements were carried out both at CINaM in Marseille and at ISM in Rome using the Scienta Omicron STM systems operating in ultra-high vacuum (UHV) (base pressure $10^{-10}$ Torr) at $\mathrm{T}=77 \mathrm{~K}$ or at room temperature (RT), respectively. No significant differences were observed by probing full or empty states in the applied bias range, or by modifying the tunneling current or measurement temperature. The STM images were successfully replicated independently in the two laboratories, demonstrating the robustness of the measurements. Sample cleaning was achieved by repeated cycles of $\mathrm{Ar}^{+}$sputtering and annealing at $780 \mathrm{~K}$. Si was evaporated from a direct current heated piece of silicon wafer kept at $1520 \mathrm{~K}$, after preoutgassing at $1070 \mathrm{~K}$ for several hours, on the Ag substrate maintained at $\sim 483$ $\mathrm{K}$. The Si flux was $\sim 1.5$ monolayer (ML)/h, where $1 \mathrm{ML}$ corresponds to the $\operatorname{Ag}(110)$ surface atom density. All images were subsequently calibrated using atomically resolved images of the 
$(5 \times 2) / c(10 \times 2)$ Si superstructure with an accuracy of $\pm 5 \%$. The lattice parameters of $\mathrm{Ag}(110)$ are $a_{A g}$ in the [001] direction and $a_{A g} / \sqrt{ } 2$ in the [110] direction where $a_{A g}=4.085 \AA$. The STM data were processed using Gwyddion software.$^{41}$ Standard plane subtraction, 1D fast Fourier transform (FFT) filter and punctually Gaussian averaging were applied to the images.

GIXD. GIXD experiments were performed at the SIXS beamline of SOLEIL synchrotron. The $\mathrm{Ag}(110)$ sample was prepared by repeated cycles of Ar+ sputtering and annealing at $\mathrm{T}=780 \mathrm{~K}$. Si was evaporated in the diffraction chamber from a Si rod using a commercial Scienta Omicron ebeam evaporator with a sample kept at $\sim 483 \mathrm{~K}$. The Si flux was kept constant during evaporation with deposition rate of $\sim 2 \mathrm{ML} / \mathrm{h}$. The sample was analyzed with $18.46 \mathrm{keV}$ x-rays at a grazing incidence angle of $0.2^{\circ}$. The diffracted x-rays were detected by an Adaptive Dynamics hybrid pixel detector. ${ }^{42}$ Diffracted intensity was measured by performing rocking scans around diffraction conditions. We used the "binoculars" software to produce three-dimensional (3D) intensity data in the reciprocal space from the raw data. ${ }^{43}$ The intensity was further integrated along the direction parallel to the surface to get the structure factors. For this purpose, the data were fitted with the product of a Lorentzian lineshape with a Gaussian lineshape, using a home-made software.

In the STM and GIXD chambers, the substrate temperature was carefully determined by calibrating the sample heater with a thermocouple. The accuracy of the temperature measurement is $\pm 10 \mathrm{~K}$. The substrate temperature during $\mathrm{Si}$ deposition $(483 \mathrm{~K})$ was chosen to avoid the formation of the massive nanostructures called nanodikes and nanotrenches that start to appear above $490 \mathrm{~K}$ and completely replace the pentamer chain phase at $550 \mathrm{~K} .{ }^{44}$ As shown in Figure $\mathrm{S} 1$ (Supporting Information), the LEED patterns acquired in the different set-ups and associated with the Si reconstructions presented here are very similar. 
Theory. DFT calculations were performed within a plane wave and norm-conserving pseudopotential framework using Quantum-ESPRESSO. ${ }^{45}$ The local density approximation (LDA) was used as in previous successful studies on $\mathrm{Si} / \mathrm{Ag}(110)$ and $\mathrm{Si} / \mathrm{Ag}(111) .^{31,46,47}$ The kinetic energy cutoff was $30 \mathrm{Ry}$. The theoretical Ag lattice constant of $4.075 \AA$ A was used (experiment: $4.085 \AA$ Å). Si/Ag slabs were modeled using asymmetric slabs containing 5 layers of Ag whose backmost two layers were fixed to the bulk positions. Structural relaxations followed a tight force threshold of $2.5 \mathrm{meV} / \mathrm{A}$. Gaussian smearing $(0.04 \mathrm{eV})$ was used to treat metallic occupancy. Supercells of size $(13 \times 4), c(18 \times 4),(8 \times 4),(5 \times 4)$, and $c(8 \times 4)$ were used along with $\Gamma$-centred $\boldsymbol{k}$ point grids of $(2 \times 8),(2 \times 8),(4 \times 8),(8 \times 8)$, and $(8 \times 8)$, respectively. These yield formation energies converged to $\sim 1 \mathrm{meV} /(1 \times 1)$ cell. The pentamer chain reconstruction was modeled with $6 \mathrm{Ag}$ layers (to accommodate the missing Ag row) in a $(5 \times 4)$ unit cell. Constant-current STM images were simulated using the Tersoff-Hamann approach. ${ }^{48}$ The surface formation energy $\gamma$ of each Si/Ag slab of area $A$ containing $N_{S i}\left(N_{A g}\right)$ atoms of $\mathrm{Si}(\mathrm{Ag})$ was computed with respect to the Si chemical potential $\mu_{S i}$ using $\gamma=\left[E_{S i / A g}\left(N_{A g}, N_{S i}\right)-N_{A g} E_{A g}^{b u l k}-N_{S i} \mu_{S i}\right] / A$.

Here $E_{S i / A g}$ is the total energy of the (relaxed) $\mathrm{Si} / \mathrm{Ag}$ heterostructure, and $E_{A g}^{b u l k}$ is the energy of a $\mathrm{Ag}$ atom in the bulk. With the exception of the pentamer chain model, the number of Ag atoms $N_{A g}$ is the same in each case. Relative formation energies with respect to that of the clean $\operatorname{Ag}(110)$ surface were then computed for each cell size. In this way, errors related to inequivalent $\boldsymbol{k}$-point sampling along the [001] direction in different cells were minimized via cancellation. 


\section{RESULTS}

\section{STM measurements}

We start the experiments by preparing the well-known Si NR superstructure obtained upon $\mathrm{Si}$ deposition on $\operatorname{Ag}(110)$ held at $483 \mathrm{~K}$ (see ${ }^{49,50,51}$ for the earliest articles). This $2 \mathrm{D}$ layer is composed of double-strand nanoribbons (DNRs) arranged in an extended $(5 \times 2) / \mathrm{c}(10 \times 2)$ superstructure. ${ }^{44,52}$ It has been demonstrated that these DNRs correspond to twin Si pentamer chains with $12 \mathrm{Si}$ atoms per $5 \times 2$ unit cell (see Figure S4g in the Supporting Information). ${ }^{30}$ This atomistic model was confirmed by combining GIXD measurements with STM imaging and DFT formation energy calculations and also supported by a combined high-resolution noncontact atomic force microscopy (nc-AFM) and tip-enhanced Raman spectroscopy (TERS) study ${ }^{46,53}$ After completion of the $(5 \times 2) / \mathrm{c}(10 \times 2) \mathrm{Si}$ overlayer, additional $\mathrm{Si}$ deposition at the same temperature leads to the progressive transformation of the $(5 \times 2) / \mathrm{c}(10 \times 2)$ domains into new $2 \mathrm{D}$ structures. As described in the Supporting Information (see Figure S2), our STM observations strongly suggest that during this process the $\operatorname{MR~} \operatorname{Ag}(110)$ reconstruction associated with the $(5 \times 2) / c(10 \times 2)$ structure is lifted and the new structures grow on an unreconstructed $\operatorname{Ag}(110)$ surface. It should be noted that these structures appear as nanostripes running along the [110] direction, i.e, parallel to the initial pentamer chains. Thus, the pentamer chain structure acts as a guide for the growth of the new structures. Even if the nanostripes do not all have the same pattern, they all appear as a beaded surface (in blue in Figure 1) with some bright protrusions (in yellow in Figure 1) on top of it, forming locally ordered domains all having a $\times 4$ periodicity along the $[1 \overline{1} 0]$ direction. Considering their relative apparent height above the other atoms $(\sim 0.7 \AA)$, these bright protrusions can be assigned, as demonstrated later, to the adsorption of extra atoms on top of the grown nanostripes and they will be thus denoted adatoms hereafter. As can be seen in Figure 1, the adatoms do not 
randomly cover the surface. Firstly, they almost exclusively form pairs (less than $10 \%$ of the adatoms are isolated); secondly, the surface separates into domains where the pairs of adatoms have formed ordered motifs, and domains free of adatoms.

In domains free of adatoms, the nanostripes display two different widths associated with three different motifs (see Figure 1 and 2). A "Ladder" motif is associated with a stripe width of $4 a_{A g}$ or $5 a_{A g}($ denoted hereafter Ladder $\times 4$ and Ladder $\times 5)$. The high-resolution STM image displayed in Figure 2 a shows that the Ladder $\times 4$ and Ladder $\times 5$ motifs have a similar structure. They show alternating groups of two moderately bright protrusions along the [1ㅣ] direction. They differ by a line of three protrusions that appears on the right side of the Ladder $\times 5$ motif, resulting in an extra p2 symmetry axis in the unit cell. The STM images resemble some models of zigzag silicene NRs on $\mathrm{Ag}(110)$ predicted by DFT. ${ }^{28,47}$ A second kind of adatom-free domain is shown in the highresolution STM image in Figure 2b, characterized by an octagon motif and by a width of $4 a_{A g}$ (denoted hereafter Octagon). In these adatom-free domains, consecutive Ladder $\times 5$ nanostripes are never found, while alternating Ladder $\times 4$ and Ladder $\times 5$ nanostripes are often found. At first glance, these STM images suggest an adlayer reconstruction composed of wide Si stripes or NRs. However, we note here that, taking $a_{A g}=4.085 \AA$ and $a_{S i}=3.847 \AA$ for silicene (see Table $\mathrm{S} 1$ and Figure S3 in the Supporting Information), there is good matching for silicene $(5 \times 3)$ reconstruction on $\operatorname{Ag}(4 \times 4)$ (misfit of $1.9 \%)$ and silicene $(6 \times 3)$ on $\operatorname{Ag}(5 \times 4)$ (misfit of $-2.1 \%$ ), as well as excellent matching for silicene $(11 \times 3)$ on $\operatorname{Ag}(9 \times 4)$ (misfit of $-0.3 \%)$ and $(16 \times 3)$ on $\operatorname{Ag}(13 \times 4)$ (misfit of $0.4 \%)$. As it will be demonstrated below, the observed structures indeed correspond to an epitaxial silicene layer on unreconstructed $\operatorname{Ag}(110)$.

Concerning the domains covered with adatoms, if one assumes that the Ladder and Octagon motifs correspond to a honeycomb organization of Si atoms, the observed bright features would 
correspond to Si adatoms on top of a silicene plane. Indeed, such silicene+adatom sheets have been first theoretically hypothesized for free-standing silicene and silicene on $\operatorname{Ag}(111){ }^{32,39}$ It has been proposed that, similar to $\mathrm{Si}$ adatoms on top of the $(7 \times 7)$ reconstruction of $\mathrm{Si}(111)$, adatoms form a DB configuration in which two $\mathrm{Si}$ atoms are vertically aligned and equidistant from the mean silicene plane. Interestingly, it has been shown that the formation of DBs is stabilizing for silicene. In principle, it cannot be excluded that these adatoms may correspond to Ag adatoms, as it has been shown for Si growth on $\operatorname{Ag}(111)$ that Ag plays the role of a surfactant layer. ${ }^{54}$ However, because of the low diffusion barriers computed for $\mathrm{Ag}$ on $\mathrm{Si}(111),{ }^{55}$ one expects a very high mobility of Ag adatoms on a silicene sheet, which would be in contradiction with the observation of very stable STM images of the adatoms-covered domains at room temperature. We anticipate here that this evidence will be confirmed by DFT calculations (see Figure 4b) reported in the following section.

As illustrated in Figure 1, the pairs of adatoms are associated to local ordered reconstructions: a c $(8 \times 4)$ reconstruction with 4 adatoms per unit cell (denoted $c(8 \times 4)+4$ adatoms hereafter), already reported by some of us, ${ }^{44}$ a $(13 \times 4)$ reconstruction with 4 adatoms per unit cell (hereafter denoted $(13 \times 4)+4$ adatoms $)$, and a $c(18 \times 4)$ reconstruction with 4 adatoms per unit cell (hereafter denoted $c(18 \times 4)+4$ adatoms $)$. The presence of a $13 \times 4$ phase is interesting and not unexpected, given the excellent matching between silicene $(16 \times 3)$ on $\operatorname{Ag}(13 \times 4)$. Figure $2 \mathrm{c}$ suggests that the $(13 \times 4)+4$ adatoms structure corresponds to Ladder motifs covered with adatoms. From Figure 1 , it seems that this is also the case for the $c(18 \times 4)+4$ adatoms structure. Finally, the adatom pattern that covers the $c(8 \times 4)+4$ adatoms structure makes it difficult to resolve the atomic structure of the underlying layer (see Figure 2d). 
To demonstrate the formation of silicene on $\operatorname{Ag}(110)$ and to precisely determine the atomic configurations of the different observed reconstructions, it is however necessary to compare the STM results with DFT simulations and to perform quantitative surface diffraction measurements.
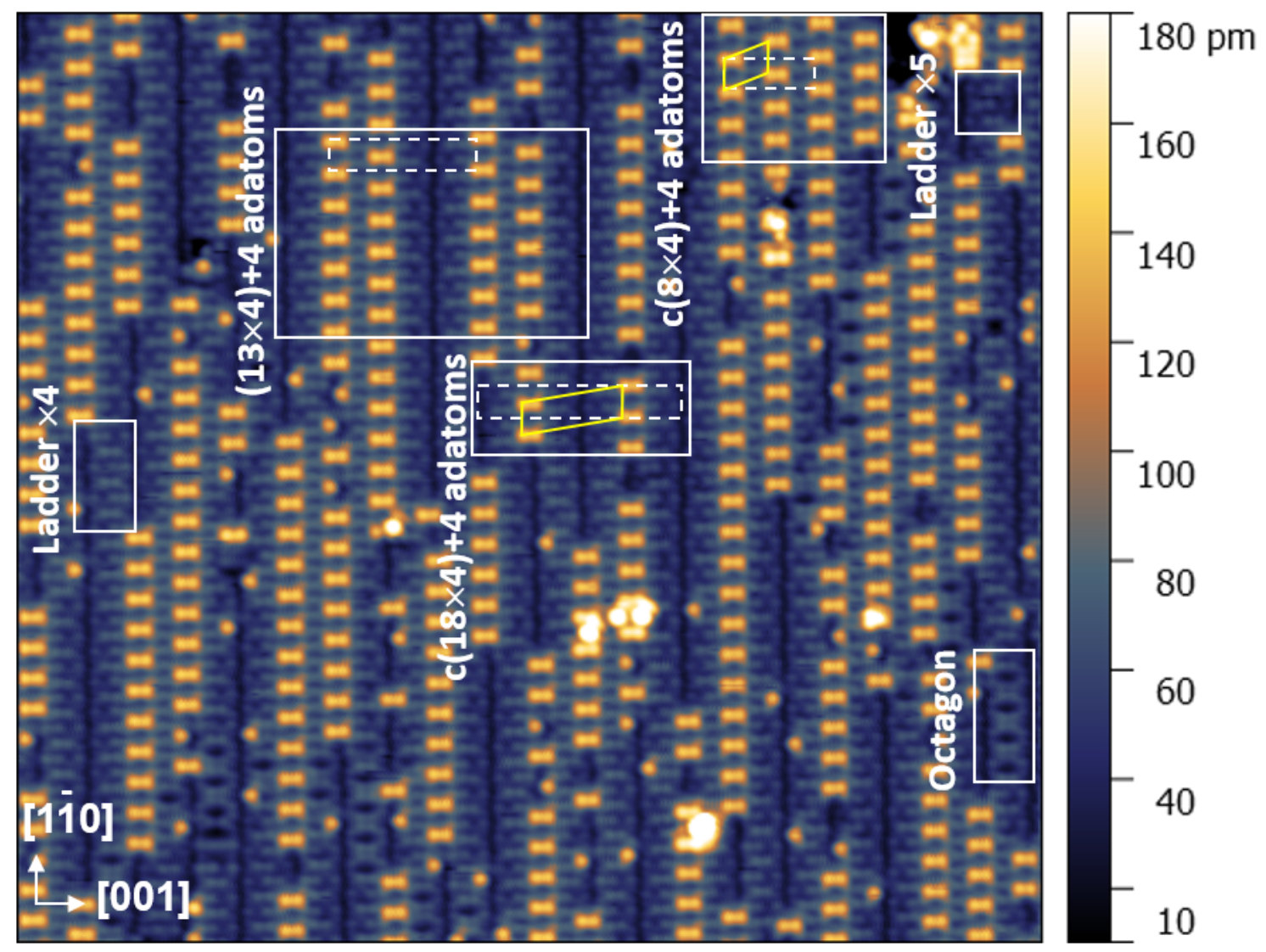

Figure 1. STM image $(77 \mathrm{~K}),(370 \times 330) \AA^{2}$, of the $\operatorname{Ag}(110)$ substrate after Si deposition at 483 $\mathrm{K}$, above completion of the pentamer chain overlayer. Ladder $\times 4$, Ladder $\times 5$ and Octagon motifs are observed in domains free of adatoms, while $c(18 \times 4)+4$ adatoms, $(13 \times 4)+4$ adatoms and $c(8 \times 4)+4 a d a t o m s$ structures are observed on the rest of the surface (the white dashed rectangles highlight the unit cells). The primitive unit cell of the $c(8 \times 4)+4$ adatoms and $c(18 \times 4)+4$ adatoms structures are indicated by yellow parallelograms. $\mathrm{I}=20 \mathrm{pA}, \mathrm{V}_{\text {sample }}=100 \mathrm{mV}$. 

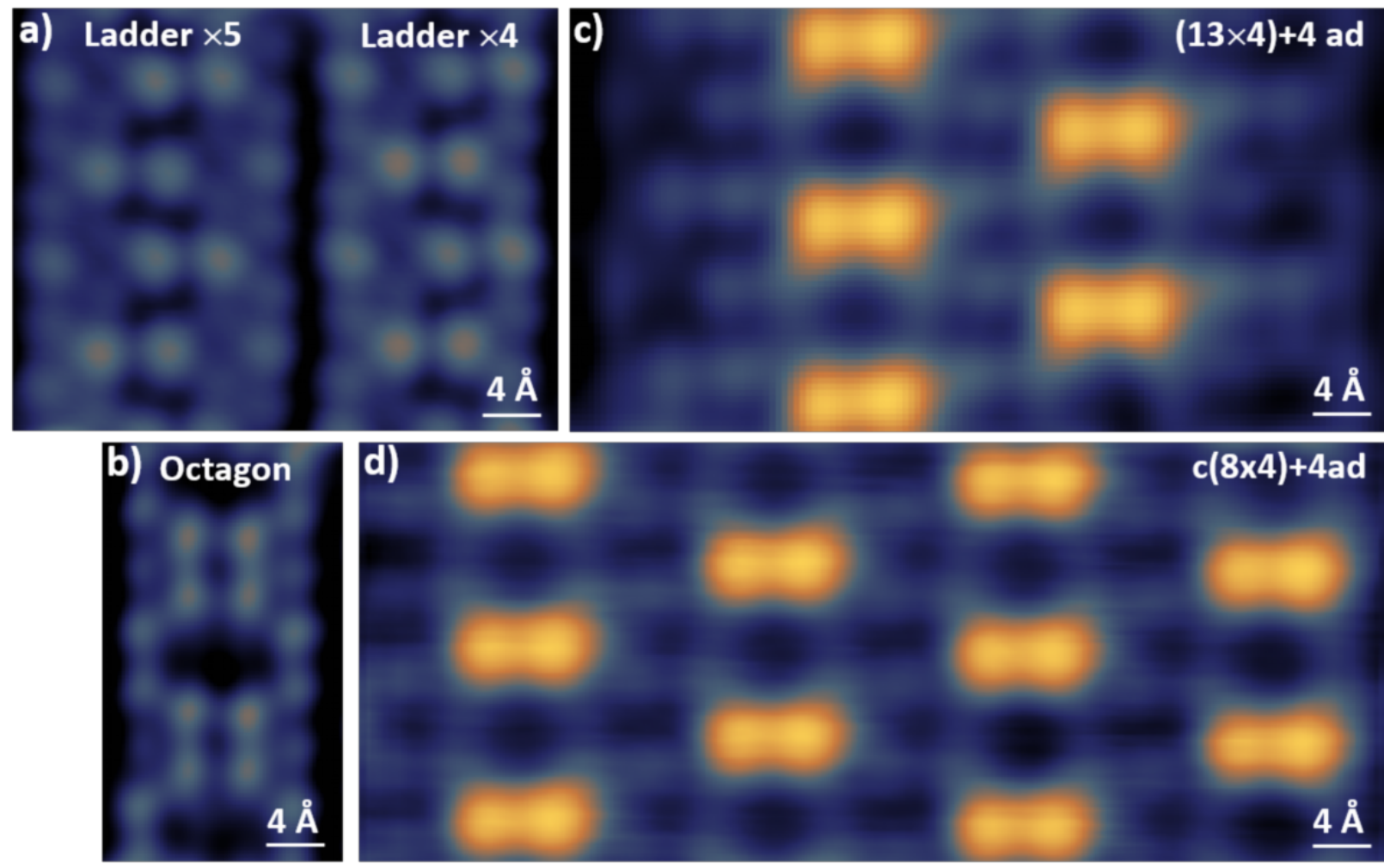

Figure 2. High-resolution STM images of adatom free motifs: (a) Ladder $\times 5$ and Ladder $\times 4$ motifs $\left(\mathrm{I}=100 \mathrm{pA}, \mathrm{V}_{\text {sample }}=100 \mathrm{mV}\right),(\mathrm{b})$ Octagon motif $\left(\mathrm{I}=5 \mathrm{nA}, \mathrm{V}_{\text {sample }}=200 \mathrm{mV}\right)$. High-resolution STM images of adatom-covered structures: (c) $(13 \times 4)+4$ adatoms structure $\left(I=460 \mathrm{pA}, \mathrm{V}_{\text {sample }}=\right.$ $-570 \mathrm{mV}),(\mathrm{d}) \mathrm{c}(8 \times 4)+4$ adatoms structure $\left(\mathrm{I}=460 \mathrm{pA}, \mathrm{V}_{\text {sample }}=-570 \mathrm{mV}\right)$. The STM images were acquired at $77 \mathrm{~K}$ in (a), (c), (d) and at RT in (b).

\section{DFT simulations}

The STM measurements reveal a variety of surface reconstructions with apparently different periodicities along [001], making atomistic simulations difficult. Guided by the aforementioned matching conditions along both [001] and [110] as well as the presence of distinct $\mathrm{c}(8 \times 4), \mathrm{c}(18 \times 4)$, and $(13 \times 4)$ domains in the STM images, we performed DFT calculations of periodic silicene adlayers placed atop $\mathrm{c}(8 \times 4), \mathrm{c}(18 \times 4)$, and $(13 \times 4)$ slabs of bulk-truncated $\mathrm{Ag}(110)$. Due to their number and size, we report the full set of models in Figure S4 (Supporting Information). They 
correspond to Si coverages of $1.83-1.88$ monolayers (ML) in $\mathrm{Ag}(110)$ surface atom density. By varying the in-plane offset of the silicene layer on the $\operatorname{Ag}(110)$ substrate, we obtained, following geometry optimization, the variety of stable structural models shown. For comparison, we also consider the pentamer double nanoribbons (P-DNR) model (1.2 ML Si) that forms on a MR Ag substrate, ${ }^{30}$ and a silicene-like nanoribbon $(5 \times 4)$-Si NR (1.4 ML Si) discussed elsewhere. ${ }^{28,47}$

Our STM simulations for silicene on $\operatorname{Ag}(110)$ reveal the presence of two periodic motifs common to all structural models (Figure S4 in the Supporting Information). These "Ladder" and "Octagon" motifs span 4 or 5 Ag lattice constants along the [001] direction, depending on the cell size, and clearly echo the experimental findings. In Figure 3a-c we compare simulated and measured STM images for typical motifs in adatom-free domains. The agreement is excellent. Both Ladder and Octagon motifs are found to arise from Si atoms that are buckled upwards due to their position atop Ag atoms. Dark lines along [1ํㅣㅇ are revealed to be due to flat Si zigzag chains lying in the shallow $\operatorname{Ag}(110)$ troughs (see black arrows). Thus, the apparent 1D nanopattern observed by STM is due to local alignments of the continuous silicene layer with the atomically corrugated $\operatorname{Ag}(110)$ surface. The intensity of the corrugation alongside the troughs is also well reproduced in the calculations. Note that although isolated NRs (see Figure S4h in the Supporting Information) also naturally produce dark lines, their widths are considerably larger than those observed experimentally and theoretical STM images of these NRs do not reproduce the experimental observations. On the contrary, the silicene models reproduce all features observed. In particular, the high-resolution image in Figure $3 \mathrm{~b}$ also reveals weaker spots among the rungs of the Ladder motif. These are well explained by the DFT simulations as arising from lower-lying Si atoms in the buckled silicene sheet. The calculations also suggest two kinds of Ladder structure: besides the symmetric motifs shown in Figure 3 a, we also identify asymmetric Ladder $\times 5$ motifs 
as indicated in Figure $3 \mathrm{c}$ by crooked lines. It has to be noted that the observation of these patterns in STM images is based on the presence of a slight contrast between Si atoms inside the Ladder motif, making them difficult to resolve.

These results strongly suggest the presence of a complete silicene sheet on the $\operatorname{Ag}(110)$ surface at high Si coverages $(1.83-1.88 \mathrm{ML})$. As discussed above, the STM measurements in Figure 1 also reveal a rich tapestry of adatom-decorated domains. For epitaxial silicene, one expects that these adatoms preferentially adsorb on top of $\mathrm{Si}$ atoms that are already below the mean silicene plane, i.e. those located in four-fold hollow site of the $\operatorname{Ag}(110)$ lattice (see Figure 3f). Theoretical studies on free-standing silicene have shown that the pairs of adatoms located at opposite ends of the hexagon ring yield a stable geometry.$^{56} \mathrm{We}$ thus considered gradual increase in adatom density via addition of adatom pairs from the lowest coverage possible in our simulations ( 2 adatoms per $(13 \times 4)$ cell) to the highest observed experimentally ( 4 adatoms per $c(8 \times 4)$ cell). For the sake of completeness, we have also computed similar structures where Ag adatoms replace Si adatoms.

Optimized geometries for the various models studied are shown in Figure S5 (Supporting Information). The presence of adatom pairs reduces the number of possible silicene geometries because they indeed lock the DBs into the four-fold hollow sites of $\operatorname{Ag}(110)$ (see Figure 3f). Simulated STM images are compared in Figure 3d and 3e with high-resolution experimental scans of the $(13 \times 4)$ and $c(8 \times 4)$ domains. The agreement is again excellent, providing a clear confirmation that the pair of bright spots derive from adatom pairs on the silicene sheet forming DBs above the $\operatorname{Ag}(110)$ hollow sites. Underneath the adatom layer, the simulation shows that silicon atoms appear to align in Ladder formation rather than as Octagons. We note here that, from the STM simulations alone, it is not possible to determine the chemical nature of the adatoms: compare for instance 
Figure S5i and S5j in the Supporting Information). However, in the following we will demonstrate that models with $\mathrm{Ag}$ adatoms are energetically unfavored.

Final confirmation of our proposed models is provided by considering their thermodynamic stability. For each model we calculated the surface formation energy and compared it with those of the bare $\operatorname{Ag}(110)$ substrate and the pentamer DNR model (P-DNR, Figure S4g in the Supporting Information) known to be stable at low Si coverages. Results for the adatom-free models are shown in Figure 4a and demonstrate that all models of silicene on $\operatorname{Ag}(110)$ become more favorable than P-DNR above a certain Si chemical potential (higher Si coverage). A quasi-degeneracy is found: the energy differences between the different models are very small $(<7 \mathrm{meV} /(1 \times 1)$ cell $)$ and close to the precision of the calculations $(\sim 1 \mathrm{meV} /(1 \times 1)$ cell $)$. The larger $\mathrm{c}(18 \times 4)$ and $(13 \times 4)$ models containing Ladder motifs appear slightly more stable, in alignment with the superior lattice matching demonstrated above for these cells, and correctly reflecting the relative paucity of Octagon motifs seen experimentally. The results imply that several silicene phases could coexist on the surface under Si-rich conditions. In contrast, Figure 4a confirms that broken NRs of silicene [(5×4)-Si NR structure, Figure S4h in the Supporting Information] are completely unfavored. Our calculations also show that the MR reconstruction of $\operatorname{Ag}(110)$, as found in the P-DNR model, is energetically unfavored to support an unbroken silicene sheet and also leads to a strongly corrugated overlayer and STM pattern [(5×4)-MR-Si structure, Figure S4i in the Supporting Information].

The phase diagram for adatom-decorated phases is reported in Figure 4b. Comparison with Figure 4a shows, above a certain Si chemical potential, a clear gain in energy for the adatomdecorated phases with respect to the adatom-free phases (delimited by the shaded regions). There is also a well-defined increase in stability as the adatom coverage increases. The most stable 
reconstruction overall is the adatom-covered $\mathrm{c}(8 \times 4)$ phase on a Ladder-reconstructed silicene sheet [c(8×4)-L+4adatoms]. In fact, for each cell size, models based on Ladders are more stable, in agreement with the STM observations (Figure 1 and 2c,d). Models with Ag adatoms (grey lines on Figure $4 \mathrm{~b}$ ) instead of Si are proven to be thermodynamically unstable and can be definitively ruled out. It is instructive to track the change in formation energy (at fixed $\mu_{\mathrm{si}}$ ) for the most stable phase of each cell size as a function of adatom coverage, as shown in Figure 4c. As the coverage (per unit cell) increases, the most stable phase alternates between $\mathrm{c}(18 \times 4)$ and $13 \times 4$ before finally stabilizing at $c(8 \times 4)$. Curiously, adding adatoms to the $13 \times 4$ cell does not result in a monotonic (negative) increase of formation energy. In fact, for coverages above 0.077 adatoms $/(1 \times 1)$ cell, the diagram suggests a phase separation into local $c(8 \times 4)+4$ adatoms and $(13 \times 4)+4$ adatoms domains, as indicated by the dotted line. Nonetheless, the difference in energy between the various models is very small, and again supports a co-existence of phases. The sample region shown in Figure 1, for example, has a mean coverage of 0.064 adatoms $/(1 \times 1)$ cell (neglecting isolated adatoms). According to Figure $4 c$, this predicts formation of $c(18 \times 4)$-L+4adatoms and $(13 \times 4)$-L+4adatoms structures, both of which are specifically found in ordered patterns in Figure 1. 


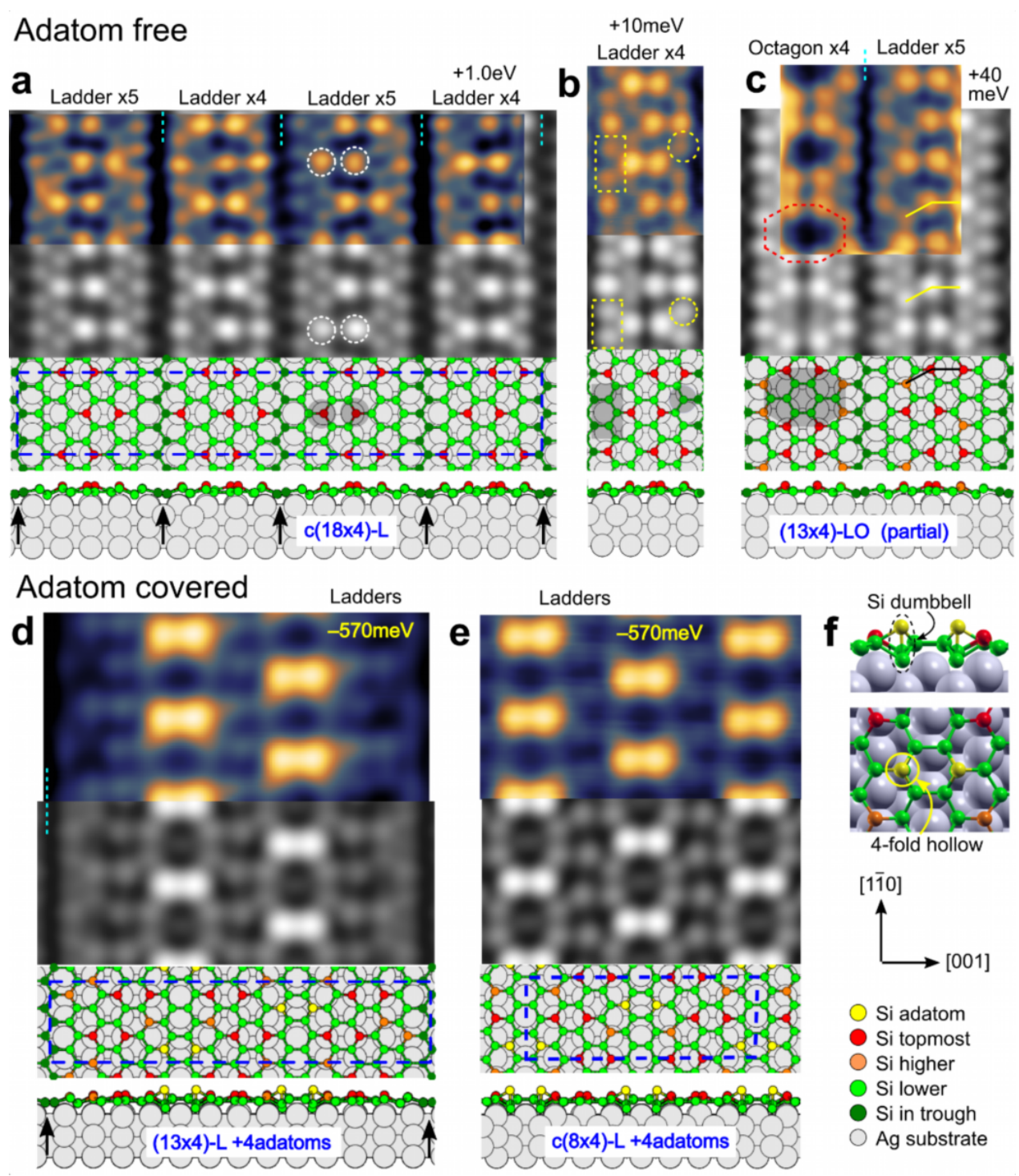

Figure 3. Experimental (blue-yellow colormap, top) and simulated (greyscale, center) STM images and corresponding structural models (bottom). Simulated images are computed at the experimental bias. The contrast of the experimental STM images has been magnified with respect to Figure 2. (a) c $(18 \times 4)$ phase showing Ladder motifs. Dotted circles indicate a "rung" due to pairs of up-buckled Si atoms. (b) A high-resolution image of a Ladder $\times 4$ region. Dotted features indicate weaker spots from lower Si atoms. (c) Partial $(13 \times 4)$ image showing the Octagon motif alongside a Ladder structure. Note the presence of asymmetric features indicated by crooked lines. (d) Full (13×4) image with 4 adatoms per cell. (e) $\mathrm{c}(8 \times 4)$ pattern at maximum adatom coverage $(4$ per cell). (f) Local adatom geometry showing DB pair formation at 4-fold hollow site. Unit cells 
are indicated in each panel. The experimental STM images were acquired at $77 \mathrm{~K}$ in (b), (c), (d), (e) and at RT in (a).
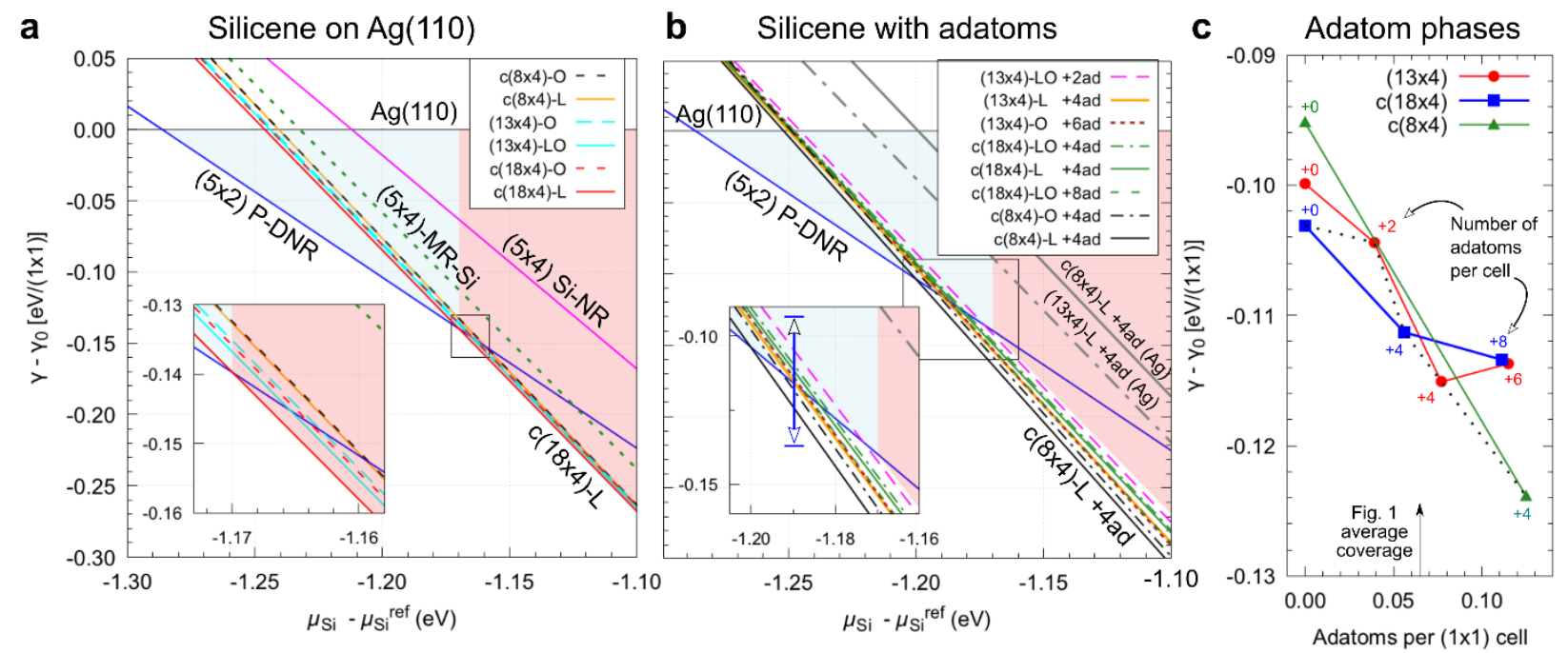

Figure 4. Phase diagram of silicene reconstructions on $\mathrm{Ag}(110)$. See Figure S4 and S5 (Supporting Information) for a full explanation of each geometry. Formation energy $\gamma$ is plotted with respect to that of clean $\operatorname{Ag}(110)$. The Si chemical potential $\mu$ is given with respect to a suitable reference value, here taken to be the average energy per atom in an isolated Si pentamer nanoribbon. (a) Pure silicene phases. The range of stability of the pentamer double nanoribbon phase (P-DNR) is indicated by the left shaded area. (b) Silicene phases decorated with adatoms. (c) Dependence of formation energy on adatom coverage for the most stable phase of each cell size at a typical value of $\Delta \mu=-1.19 \mathrm{eV}$. Energy range is indicated by the blue double arrow within inset of (b). The pathway that maximizes the formation energy for increasing coverage is indicated by dotted lines.

\section{GIXD measurements}

Confirmation of the long range ordered geometry of the silicene overlayer is provided by GIXD measurements. GIXD is indeed a powerful technique for the discrimination of superstructure models and for the refinement of atomic positions. It has been used to confirm silicene growth on $\operatorname{Ag}(111)$ and precisely determine the atomic positions in the unit cells of the 
various superstructures formed. ${ }^{57,58}$ The $(h, k, l)$ indices used for indexing a reflection in reciprocal space refer to the $\operatorname{Ag}(110)$ surface basis $\left(a_{A g}=4.085 \AA, b_{A g}=2.889 \AA, c_{A g}=2.889 \AA, \alpha=\beta=\right.$ $\left.\gamma=90^{\circ}\right)$. During Si evaporation at $\mathrm{T}=483 \mathrm{~K}$, we have followed the evolution of the diffracted intensity along the $h$ direction at $(k=0, l=0.1)$. The corresponding scans are shown in Figure 5a. The peak at $h=1$ observed before evaporation corresponds to the crystal truncation rods. As soon as evaporation starts, diffraction peaks at $h=n / 5$ are observed which are associated with the formation of $(5 \times 2) / \mathrm{c}(10 \times 2)$ domains of Si DNRs. ${ }^{46,52}$ When the intensity of these peaks starts to decrease, another peak appears at $h=1.232 \pm 0.005 \sim 16 / 13$. The evaporation was stopped when the maximum of intensity for this peak was obtained, i.e. for around twice the evaporation time corresponding to the maximum of intensity of the $(5 \times 2) / \mathrm{c}(10 \times 2)$ peaks.

At this point, the intensity of the peaks at $h=n / 5$ ( $n$ integer) has almost completely vanished. This indicates that the new structure does not form on top of the Si pentamers of the DNRs, but that it replaces them. Moreover, it has been shown (see ${ }^{46}$ ) that the MR reconstruction of the substrate significantly contributes to the intensity of the $h=n / 5$ peaks. The vanishing of these peaks demonstrates that the substrate MR reconstruction is lifted. This conclusion is in agreement with the growth mechanism suggested by the STM observations of coexisting $(5 \times 2) / \mathrm{c}(10 \times 2)$ and silicene phase domains (see Figure S2 in the Supporting Information). Assuming that the maximum of intensity of the peak at $h=1.4$ corresponds to $1.2 \mathrm{ML}$, the reconstruction measured would correspond to $2.4 \mathrm{ML}$. This is higher than the coverage range [1.83-1.88 ML] of the silicene and DB silicene structures computed by DFT. However, we cannot exclude the fact that the second structure starts to form before completion of the first one, or that the Si sticking coefficient is not the same for both structures. 
After the Si deposition, structure factors were measured for both in-plane and out-of-plane conditions. All measured structure factors could be indexed on the basis of a $(13 \times 4) \operatorname{Ag}(110)$ reconstruction. The full width at half maximum (FWHM) of the peak at $\left(\frac{16}{13}, 0,0.1\right)$ is $\Delta h=0.023$. Note that this small value excludes the fact that the signal may arise from the superimposition of diffraction from $\mathrm{c}(18 \times 4)$ and $\mathrm{c}(8 \times 4)$ domains, since they would correspond to peaks at $h=1.222$ and $h=1.25$, which are separated by $\Delta h=0.028$. However, we cannot totally exclude the presence of a low density of $c(18 \times 4)$ and $c(8 \times 4)$ domains, having a small size. The FWHM of the peaks is given by the size of the domains and the dispersion of the lattice parameter $\Delta a / a$. From the different peaks, we estimate the domain size to be $400 \AA$ and the lattice parameter dispersion to be 0.01 . Along $k$, the FWHM is smaller (for example, $\Delta k=0.008$ for the $\left(0, \frac{6}{4}, 0.1\right)$ peak) and other contributions to the FWHM, such as the sample size, cannot be neglected.

In the following, the $(H, K, L)$ indices refer to the $(13 \times 4) \operatorname{Ag}(110)$ reconstruction $\left(a_{13 \times 4}=53.105 \AA, b_{13 \times 4}=11.556 \AA, c_{A g}=2.889 \AA, \alpha=\beta=\gamma=90^{\circ}\right)$. For in-plane conditions, excluding substrate spots, significant intensity was only detected for a few set of values, namely for $(H, K)=(16 n+8 p, p)$ (see Figure $5 b)$. From the corresponding measured in-plane structure factors, we have computed the 2D Patterson map, shown in Figure 5c, which is the electron density-density autocorrelation function within the surface unit cell: ${ }^{59}$

$P(x, y)=2 \sum_{H K}|F(H, K)|^{2} \cos (2 \pi(H x+K y))$

The Patterson map is very similar to that of the honeycomb structure: the intensity, normalized to its value at the origin, is close to 1.0 at the node of the hexagonal unit cell drawn in green, and to 0.25 at the closest interatomic distance, corresponding to the correlation between half of the atoms of the unit cell. The hexagonal unit cell parameters are $a=3.837 \AA, b=3.852 \AA, \gamma=120.1^{\circ}$. 
This is very close to the silicene unit cell theoretical lattice constant $a_{\text {silicene }}=3.847 \AA$, but also to the surface unit cell of $\operatorname{Si}(111): a_{S i}=3.840 \AA$. The small distortion of the hexagonal unit cell, as compared to the one of free-standing silicene, is favored by the good lattice matching with the $\operatorname{Ag}(110)$ substrate. Importantly, we can exclude that the layer corresponds to a diamond-like thin Si film, such as the one measured on $\operatorname{Ag}(111)$, since the Patterson map would be different (see Figure S7 in the Supporting Information).$^{54}$
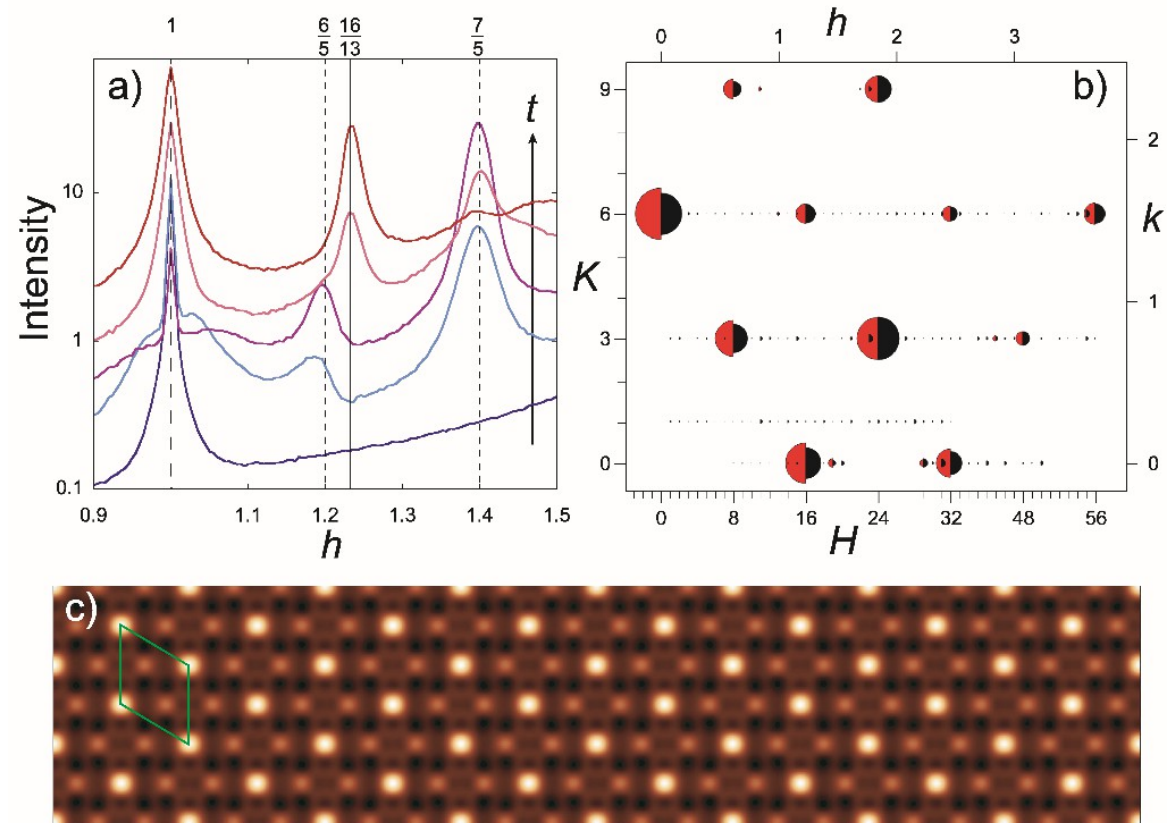

Figure 5. (a) Evolution of the diffracted intensity measured along the $h$ direction at $(k=0, l=0.1)$ during $\mathrm{Si}$ evaporation. From blue to red: scans at $t=0 \mathrm{~s}, 18 \mathrm{~min}, 36 \mathrm{~min}, 59$ $\min$ and $76 \mathrm{~min}$. The peak at $h=1$ is indicated with a dashed line, the peaks at $h=6 / 5$ and $h=7 / 5$ are indicated with a dotted line while the peak at $h=16 / 13$ is indicated with a continuous line. The maximum of intensity of the $h=7 / 5$ and $h=8 / 13$ peaks are obtained for $t=38 \mathrm{~min}$ and $t=78 \mathrm{~min}$, respectively. (b) In-plane structure factors. Comparison between experimental (red half-disks) and simulated (black half-disks) structure factors (the substrate structure factors for integer values of $h$ and $k$ have not been drawn for clarity). (c) Experimental Patterson map of the $(13 \times 4) \mathrm{Si} / \mathrm{Ag}(110)$ structure. A quasi hexagonal unit cell ( $a=3.837 \AA$, $b=3.852 \AA, \gamma=120.1^{\circ}$ ) is drawn in green. 
In order to determine the vertical positions of the atoms, we have acquired diffracted intensity along 25 superstructure rods, corresponding to 16 non-equivalent rods. In addition to the rods corresponding to the honeycomb cell $((H, K)=(16 n+8 p, p))$, measurable intensity was also obtained for satellite rods at $\Delta H= \pm 3$. This indicates that the honeycomb cell exhibits a periodic vertical modulation with a period along $\operatorname{Ag}[001]$ equal to $\frac{13}{3} \times 4.085 \AA=17.70 \AA$, in good agreement with STM observations of 3 nanostripes per $(13 \times 4)$ unit cell. The rods display weak modulations, excluding the presence of several Si layers. However, the modulations measured show that all atoms are not at the same $z$ value. Moreover, the rod profiles display strong differences from one rod to another, which indicates that the layer buckling cannot correspond to that of free-standing silicene. Indeed, in the latter case, the modulation of the different rods should be similar.

We have compared the measured structure factors to theoretical structure factors simulated from various atomic configurations relaxed by DFT calculations. For this purpose, we have used only 7 free parameters: a scale factor and two sets of Debye-Waller factors for $\mathrm{Si}$ and $\mathrm{Ag}$ atoms along the $H, K$, and $L$ directions. The agreement between experimental $\left(F_{\mathrm{exp}}\right)$ and simulated $\left(F_{\mathrm{th}}\right)$ structure factors is estimated by the value of $\chi^{2}=\frac{1}{N_{\mathrm{pts}}-N_{\mathrm{par}}} \sum_{N_{\mathrm{Pts}}}\left(\frac{F_{\mathrm{th}}-F_{\mathrm{exp}}}{\sigma_{\mathrm{exp}}}\right)^{2}$ where $N_{\mathrm{pts}}$ is the number of experimental structure factors, $N_{\mathrm{par}}$ is the number of free parameters and $\sigma_{\exp }$ is the experimental uncertainty, which takes into account the statistical uncertainty given by the number of counted photons and an overall $10 \%$ uncertainty. We have first tested the most stable $(13 \times 4)$ configurations identified by DFT. Whereas a good agreement is already obtained with the two adatom-free silicene configurations tested, Figure S8 (Supporting Information) shows that the best fit is obtained for the (13×4)-L+4adatoms structure, with $\chi^{2}=4.7$. It corresponds to a R-Factor 
value $R_{F}=\frac{\sum_{N_{\mathrm{Pts}}}\left|F_{\mathrm{th}}-F_{\mathrm{exp}}\right|}{\sum_{N_{\mathrm{Pts}}} F_{\exp }}=0.19$. The comparison between experimental and simulated structure factors is shown in Figure 6 . The $(13 \times 4)$-L+4adatoms model reproduces well all variations of intensities along the diffraction rods, whereas the fit is poorer for the $(13 \times 4)$-LO configuration. The intensities of the simulated in-plane structure factors, plotted in Figure 5b, are in excellent agreement with the experimental ones. Finally, we have verified that no better agreement could be obtained with the other configurations computed by DFT and having a higher formation energy. For instance, a model of an incomplete honeycomb stripe in a $(13 \times 4)$ cell, i.e. a wider NR than that shown in Figure S4h (Supporting Information), does not correctly fit the experimental structure factors, with $\chi^{2}=19.2$.

The main and most important conclusion from this part is that GIXD demonstrates that the Si layer formed on $\operatorname{Ag}(110)$ corresponds to a DB silicene structure with a small density, equal to 0.077 adatoms per $(1 \times 1)$ cell, predicted by our DFT calculations and observed by STM. 

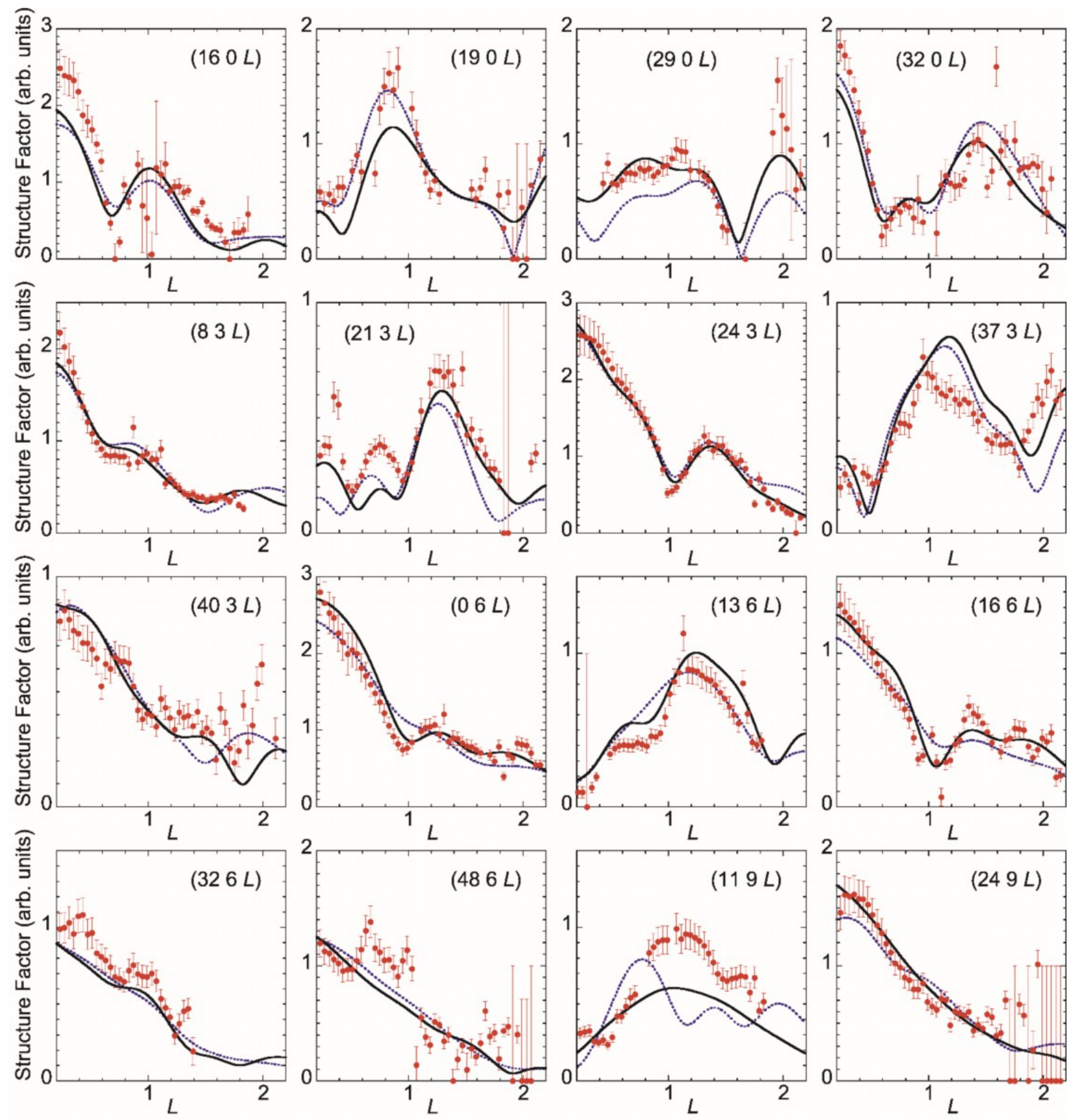

Figure 6. Structure factors for various rods of the $(13 \times 4)$ structure. Comparison between experimental (red dots) and computed values, using the $(13 \times 4)$-LO (dotted blue line) or $(13 \times 4)$ L+4adatoms (continuous black line) models simulated by DFT. 


\section{DISCUSSION}

We obtain an excellent agreement between STM observations and simulated images of silicene and DB silicene phases as well as between the DFT calculation for the $(13 \times 4) \mathrm{L}+4$ adatoms structure and the diffraction data. This demonstrates that the $\operatorname{Ag}(110)$ surface is entirely covered with a silicene layer, which is itself partially covered with adatoms in DB configurations. The ordering of the observed structures originates from both a local registry between the silicene lattice and the substrate along $\operatorname{Ag}[1 \overline{1} 0]$ with a ratio equal to $3 / 4$ and along $\operatorname{Ag}[001]$ with ratios equal to $5 / 4,11 / 9$ or $16 / 13$ for the $c(8 \times 4), c(18 \times 4)$ and $(13 \times 4)$ reconstructions, and a periodic organization of adatom pairs. Moreover, the different structures are shown to have a very similar formation energy, in agreement with the coexistence of various domains of small size observed by STM. DFT computations show that the formation energy of the silicene adlayer is lowered by adding adatoms up to a maximum density which depends on the unit cell (see Figure 4c). The simulations also show that the silicene layer is distorted to accommodate the DBs on top of four-fold hollow sites of the Ag substrate. The gain in cohesive energy from DB formation is thus balanced by the energy of deformation of the silicene layer. This is clearly visible for the $(13 \times 4)$ reconstruction for which the most favored number of adatoms is 4 per unit cell instead of 6 , implying a larger deformation penalty in the latter case. It also explains why we find a low DB density compared to the density of host sites on the silicene sheet (see the FDS or LHDS silicene phases proposed by Cahangirov et al. ${ }^{39}$ or the Si-VI phase of Borlido et al..${ }^{33}$ ), as DBs prefer to form at the fourfold hollow sites of the Ag substrate. This finding is in agreement with both STM observations and GIXD measurements. While we cannot rule out that better models of the system exist, they should only be refinements of the proposed geometries as the presence of a single-layer honeycomb geometry is demonstrated by GIXD. 
From an experimental point of view, the density of the deposited Si results from the balance between the incoming $\mathrm{Si}$ atom flux and the flux of Si atoms involved in the transformation of the pentamer NRs into silicene sheets. Ultimately, the geometry that forms locally during the Si deposition would be determined by the local coverage of Si atoms. Contrary to STM experiments, we observe only one structure by GIXD. These differences observed between STM and GIXD measurements could result from the inherently different growth conditions in the two experimental setups. In particular, a slightly higher substrate temperature, not exceeding $15 \mathrm{~K}$, may have been used for the Si deposit analysed by GIXD. Indeed, the size of the observed domains is around 400 $\AA$, significantly higher than the average domain width measured by STM, as shown in Figure 1 . As a result, the surface is better ordered with a large majority of $(13 \times 4)-\mathrm{L}+4$ adatoms domains.

It has been demonstrated that $\mathrm{Si}$ atoms strongly interact with the $\mathrm{Ag}$ substrate upon deposition, causing $\mathrm{Ag}$ atom ejection from the first layer on both the $\operatorname{Ag}(111)$ and $\operatorname{Ag}(110)$ substrates. ${ }^{52,60,61,62}$ Our GIXD measurements clearly show that the MR reconstruction is lifted when the $(5 \times 2) / c(10 \times 2)$ NR structure is converted into the honeycomb structure. It is not surprising since this MR reconstruction is intimately linked to the Si NR superstructure and becomes thermodynamically unstable when this superstructure is removed, as shown by our DFT calculations. Based on our STM observations, a mechanism is proposed, where Ag atoms of the MR reconstruction are released, leaving bare unreconstructed $\mathrm{Ag}(110)$ areas, and new $\mathrm{Ag}(110)$ terraces are concomitantly formed (see the Supporting Information). It cannot be excluded that additional Ag atom ejection occurs during the growth of the Si honeycomb structure, as observed for Si deposited on $\operatorname{Ag}(111)$ with the formation of novel $\operatorname{Ag}(111)$ terraces. ${ }^{60}$ Nevertheless, it is important to note that our GIXD experiments and DFT calculations clearly show that the Si honeycomb structure grows on an unreconstructed $\mathrm{Ag}$ substrate, as for the $\mathrm{Si} / \mathrm{Ag}(111)$ interface. ${ }^{57}$ 
We have thus demonstrated the growth of DB silicene sheets on $\operatorname{Ag}(110)$. Although quite challenging, these layers would be of great interest if decoupled from the Ag substrate. Several approaches have been reported, both experimentally and theoretically, to achieve such decoupling. For instance, first-principles calculations have shown that hydrogenation and calcium intercalation can be employed to break bonds between a silicene overlayer and a silver surface. ${ }^{63}$ A combined STM, X-ray photoemission spectroscopy (XPS), angle-resolved photoelectron spectroscopy (ARPES) and first-principles calculations study reveals that the interaction with $\operatorname{Ag}(111)$ of the top layer of a bilayer silicene can be weakened by oxygen intercalation into the Si underlayer. ${ }^{64}$ It has also been reported that an epitaxial silicene layer could be detached from the silver substrate and transferred onto target substrates. ${ }^{65,66} \mathrm{We}$ point out that the demonstrated higher stability of DB silicene compared to pristine silicene will favor the decoupling process of the DB silicene layer from the substrate. Since both pristine silicene ${ }^{2}$ as well as higher-density DB phases of silicene ${ }^{33,39}$ are reported to be dynamically stable according to phonon dispersion calculations, we expect the lower density DB phases identified here will exhibit comparable stability in their freestanding form.

The synthesis of DB silicene opens new routes to tune the peculiar properties of silicene. For instance, it has been predicted that DB configuration attributes Si coverage-dependent electronic and magnetic properties to bare silicene, such as a spin-polarized semiconducting behavior with a band gap of $\sim 80 \mathrm{meV} .{ }^{32}$ We thus performed band structure calculations to study the effect of the DBs on a free-standing layer with the atomic configurations identified in our study (see Figure S6 in the Supporting Information). As compared to the pristine free-standing silicene, the electronic structure for the $\mathrm{c}(8 \times 4)-\mathrm{L}$ reconstruction shows a small gap opening of $80 \mathrm{meV}$. Interestingly, a small gap value $(60 \mathrm{meV})$ is also obtained for the $c(8 \times 4)-L+4$ adatoms DB silicene. State 
projections onto $\mathrm{p}_{\mathrm{z}}$ orbitals and DB atoms show that $\mathrm{p}_{\mathrm{z}}$ contributions are located near the Fermi level while states localized on DB atoms give contributions at $-1.3 \mathrm{eV}$ and $-1.5 \mathrm{eV}$ below the Fermi level. Thus, DB atoms appear as good candidates for tuning the electronic properties of silicene by charge doping through the DB atom dangling bonds pointing outward from the silicene layer.

More generally, DB silicene structures open new perspectives for tailoring the chemical, physical and mechanical properties of silicene sheets with molecular attachment. Indeed, numerous experimental and theoretical works have been devoted to the reactivity of silicene towards molecular adsorption. ${ }^{67,68,69}$ For example, the adsorption of $\mathrm{NO}, \mathrm{O}_{2}, \mathrm{NH}_{3}$, and $\mathrm{SO}_{2}$ opens the band gap of silicene, rendering silicene a narrow gap semiconductor. ${ }^{69}$ The possibility of tuning the electronic properties of silicene by molecular adsorption makes silicene a promising candidate as molecular sensor. ${ }^{70,71}$ From a mechanical point of view, adsorption of $\mathrm{H}$ and $\mathrm{F}$ reduces the Young's and bulk moduli of silicene. ${ }^{72}$ Whereas a silicene sheet already presents a high reactivity, it has been shown that its reactivity is enhanced at defects sites, such as Stone-Wales defects. ${ }^{69}$ Interestingly, the high reactivity of DB silicene to hydrogen has already been noted and thus one should expect an equally strong molecular adsorption on the Si adatoms of the Si layer. ${ }^{73}$ One may expect enhanced detection performances for DB silicene deriving from specific adsorption sites.

\section{CONCLUSION}

In conclusion, our combined STM-DFT-GIXD study demonstrates the formation of silicene phases on $\operatorname{Ag}(110)$, stabilized by DBs adsorbed at four-fold hollow sites of the Ag substrate. DFT calculations show that the different structures have similar formation energies, in agreement with the coexistence of various 2D domains of small size observed by high-resolution STM. 
Nevertheless, GIXD measurements indicate that the control of the growth conditions can lead to the formation of an extended DB silicene structure whose orientation is guided by that of the $\mathrm{Si}$ pentamer chain domains initially grown on $\operatorname{Ag}(110)$. This opens up interesting perspectives for practical applications such as gas sensing and may offer a realistic platform for exploring predicted novel topological properties of DB silicene..$^{74}$ Finally, we stress that our work describes a realistic pathway for synthesizing or identifying DB-stabilized geometries on other 2D monoelemental Xene materials.

\section{ASSOCIATED CONTENT}

Supporting Information. (1) large scale STM image during growth showing mixture of pentamer chain and silicene phases, (2) mismatch study of silicene supercells on $\operatorname{Ag}(110)$, (3) DFT calculations of all Si/Ag(110) models studied in this work (geometry and STM images), (4) Band structures of silicene and dumbbell silicene, (5) Simulation of Patterson maps from GIXD, (6) Comparison between experimental and simulated structure factors for different $\mathrm{Si}$ adatom density.

\section{AUTHOR INFORMATION}

\section{Corresponding Authors}

* E-mail: laurence.masson@cinam.univ-mrs.fr

*E-mail: prevot@insp.jussieu.fr

*E-mail: conor.hogan@ism.cnr.it

\section{ORCID}

Thomas Leoni: 0000-0002-0582-9534 
Conor Hogan: 0000-0002-0870-6361

Kai Zhang: 0000-0002-6124-8793

Michel Daher Mansour: 0000-0001-6319-2650

Romain Bernard: 0000-0002-2944-142X

Romain Parret: 0000-0003-0662-6373

Andrea Resta:_0000-0002-0614-1192

Stefano Colonna: 0000-0001-9994-4287

Yves Borensztein: 0000-0002-1570-732X

Fabio Ronci: 0000-0002-7424-4046

Geoffroy Prévot: 0000-0001-7960-5587

Laurence Masson: 0000-0003-0587-2468

\section{Present Addresses}

${ }^{\perp}$ Univ. Lille, CNRS, Centrale Lille, Univ. Polytechnique Hauts-de-France, UMR 8520 - IEMN, F-59000 Lille, France

\section{Notes}

The authors declare no competing financial interest.

\section{ACKNOWLEDGMENT}

Support from the SIXS beamline staff at SOLEIL is greatly acknowledged. T. L. and L. M. thank Alain Ranguis for his valuable STM technical support at CINaM. C.H. acknowledges high performance computing resources and support from CINECA via the ISCRA initiative. K. Z. is supported by the Chinese Scholarship Council (CSC contract 201808070070).

\section{REFERENCES}


(1) Gupta, A.; Sakthivel, T.; Seal, S. Recent Development in 2D Materials beyond Graphene. Prog. Mater. Sci. 2015, 73, 44-126.

(2) Cahangirov, S.; Topsakal, M.; Aktürk, E.; Şahin, H.; Ciraci, S. Two- and One-Dimensional Honeycomb Structures of Silicon and Germanium. Phys. Rev. Lett. 2009, 102, 236804.

(3) Liu, C.-C.; Feng, W.; Yao, Y. Quantum Spin Hall Effect in Silicene and Two-Dimensional Germanium. Phys. Rev. Lett. 2011, 107, 076802.

(4) Meng, Z.; Zhuang, J.; Xu, X.; Hao, W.; Dou, S. X.; Du, Y. Electronic Band Engineering in Elemental 2D Materials. Adv. Mater. Interfaces 2018, 5, 1800749.

(5) Liu, C.-C.; Jiang, H.; Yao, Y. Low-Energy Effective Hamiltonian Involving Spin-Orbit Coupling in Silicene and Two-Dimensional Germanium and Tin. Phys. Rev. B 2011, 84, 195430.

(6) Fleurence, A.; Friedlein, R.; Ozaki, T.; Kawai, H.; Wang, Y.; Yamada-Takamura, Y. Experimental Evidence for Epitaxial Silicene on Diboride Thin Films. Phys. Rev. Lett. 2012, 108, 245501.

(7) Meng, L.; Wang, Y.; Zhang, L.; Du, S.; Wu, R.; Li, L.; Zhang, Y.; Li, G.; Zhou, H.; Hofer, W. A.; et al. Buckled Silicene Formation on $\operatorname{Ir}(111)$. Nano Lett. 2013, 13, 685-690.

(8) Aizawa, T.; Suehara, S.; Otani, S. Silicene on Zirconium Carbide (111). J. Phys. Chem. C 2014, 118, 23049-23057.

(9) Chiappe, D.; Scalise, E.; Cinquanta, E.; Grazianetti, C.; van den Broek, B.; Fanciulli, M.; Houssa, M.; Molle, A. Two-Dimensional Si Nanosheets with Local Hexagonal Structure on a $\mathrm{MoS}_{2}$ Surface. Adv. Mater. 2014, 26, 2096-2101.

(10) Huang, L.; Zhang, Y.-F.; Zhang, Y.-Y.; Xu, W.; Que, Y.; Li, E.; Pan, J.-B.; Wang, Y.-L.; Liu, Y.; Du, S.-X.; et al. Sequence of Silicon Monolayer Structures Grown on a Ru Surface: From a Herringbone Structure to Silicene. Nano Lett. 2017, 17, 1161-1166.

(11) Stpniak-Dybala, A.; Dyniec, P.; Kopciuszyski, M.; Zdyb, R.; Jałochowski, M.; Krawiec, M. Planar Silicene: A New Silicon Allotrope Epitaxially Grown by Segregation. Adv. Funct. Mater. 2019, 29, 1906053.

(12) Quertite, K.; Enriquez, H.; Trcera, N.; Tong, Y.; Bendounan, A.; Mayne, A. J.; Dujardin, G.; Lagarde, P.; El kenz, A.; Benyoussef, A.; et al. Silicene Nanoribbons on an Insulating Thin Film. Adv. Funct. Mater. 2020, 31, 2007013.

(13) Vogt, P.; De Padova, P.; Quaresima, C.; Avila, J.; Frantzeskakis, E.; Asensio, M. C.; Resta, A.; Ealet, B.; Le Lay, G. Silicene: Compelling Experimental Evidence for Graphenelike Two-Dimensional Silicon. Phys. Rev. Lett. 2012, 108, 155501.

(14) Feng, B.; Ding, Z.; Meng, S.; Yao, Y.; He, X.; Cheng, P.; Chen, L.; Wu, K. Evidence of Silicene in Honeycomb Structures of Silicon on $\operatorname{Ag}(111)$. Nano Lett. 2012, 12, 3507-3511.

(15) Lin, C.-L.; Arafune, R.; Kawahara, K.; Tsukahara, N.; Minamitani, E.; Kim, Y.; Takagi, N.; Kawai, M. Structure of Silicene Grown on Ag(111). Appl. Phys. Express 2012, 5, 045802.

(16) Jamgotchian, H.; Colignon, Y.; Hamzaoui, N.; Ealet, B.; Hoarau, J. Y.; Aufray, B.; Bibérian, J. P. Growth of Silicene Layers on $\operatorname{Ag}(111)$ : Unexpected Effect of the Substrate Temperature. J. Phys.: Condens. Matter 2012, 24, 172001.

(17) Enriquez, H.; Vizzini, S.; Kara, A.; Lalmi, B.; Oughaddou, H. Silicene Structures on Silver Surfaces. J. Phys.: Condens. Matter 2012, 24, 314211.

(18) Cahangirov, S.; Audiffred, M.; Tang, P.; Iacomino, A.; Duan, W.; Merino, G.; Rubio, A. Electronic Structure of Silicene on $\operatorname{Ag}(111)$ : Strong Hybridization Effects. Phys. Rev. B 2013, 88, 035432. 
(19) Gori, P.; Pulci, O.; Ronci, F.; Colonna, S.; Bechstedt, F. Origin of Dirac-Cone-like Features in Silicon Structures on $\mathrm{Ag}(111)$ and $\mathrm{Ag}(110)$. J. Appl. Phys. 2013, 114, 113710.

(20) Chen, M. X.; Weinert, M. Revealing the Substrate Origin of the Linear Dispersion of Silicene/Ag(111). Nano Lett. 2014, 14, 5189-5193.

(21) Mahatha, S. K.; Moras, P.; Bellini, V.; Sheverdyaeva, P. M.; Struzzi, C.; Petaccia, L.; Carbone, C. Silicene on Ag(111): A Honeycomb Lattice without Dirac Bands. Phys. Rev. B 2014, 89, 201416.

(22) Grazianetti, C.; Cinquanta, E.; Molle, A. Two-Dimensional Silicon: The Advent of Silicene. 2D Mater. 2016, 3, 012001.

(23) Son, Y.-W.; Cohen, M. L.; Louie, S. G. Energy Gaps in Graphene Nanoribbons. Phys. Rev. Lett. 2006, 97, 216803.

(24) Cai, J.; Ruffieux, P.; Jaafar, R.; Bieri, M.; Braun, T.; Blankenburg, S.; Muoth, M.; Seitsonen, A. P.; Saleh, M.; Feng, X.; et al. Atomically Precise Bottom-up Fabrication of Graphene Nanoribbons. Nature 2010, 466, 470-473.

(25) Denis, P. A. Band Gap Opening of Monolayer and Bilayer Graphene Doped with Aluminium, Silicon, Phosphorus, and Sulfur. Chem. Phys. Lett. 2010, 492, 251-257.

(26) Usachov, D.; Vilkov, O.; Grüneis, A.; Haberer, D.; Fedorov, A.; Adamchuk, V. K.; Preobrajenski, A. B.; Dudin, P.; Barinov, A.; Oehzelt, M.; et al. Nitrogen-Doped Graphene: Efficient Growth, Structure, and Electronic Properties. Nano Lett. 2011, 11, 5401-5407.

(27) Aufray, B.; Kara, A.; Vizzini, S.; Oughaddou, H.; Léandri, C.; Ealet, B.; Le Lay, G. Graphene-like Silicon Nanoribbons on Ag(110): A Possible Formation of Silicene. Appl. Phys. Lett. 2010, 96, 183102.

(28) Lian, C.; Ni, J. The Structural and Electronic Properties of Silicon Nanoribbons on Ag(110): A First Principles Study. Phys. B 2012, 407, 4695-4699.

(29) Oughaddou, H.; Enriquez, H.; Tchalala, M. R.; Yildirim, H.; Mayne, A. J.; Bendounan, A.; Dujardin, G.; Ait Ali, M.; Kara, A. Silicene, a Promising New 2D Material. Prog. Surf. Sci. 2015, 90, 46-83.

(30) Cerdá, J. I.; Sławińska, J.; Le Lay, G.; Marele, A. C.; Gómez-Rodríguez, J. M.; Dávila, M. E. Unveiling the Pentagonal Nature of Perfectly Aligned Single-and Double-Strand Si Nano-Ribbons on Ag(110). Nat. Commun. 2016, 7, 13076.

(31) Hogan, C.; Pulci, O.; Gori, P.; Bechstedt, F.; Martin, D. S.; Barritt, E. E.; Curcella, A.; Prevot, G.; Borensztein, Y. Optical Properties of Silicene, Si/Ag(111), and Si/Ag(110). Phys. Rev. B 2018, 97, 195407.

(32) Özçelik, V. O.; Ciraci, S. Local Reconstructions of Silicene Induced by Adatoms. J. Phys. Chem. C 2013, 117, 26305-26315.

(33) Borlido, P.; Rödl, C.; Marques, M. A. L.; Botti, S. The Ground State of Two-Dimensional Silicon. 2D Mater. 2018, 5, 035010.

(34) Kaltsas, D.; Tsetseris, L. Stability and Electronic Properties of Ultrathin Films of Silicon and Germanium. Phys. Chem. Chem. Phys. 2013, 15, 9710.

(35) Matusalem, F.; Marques, M.; Teles, L. K.; Bechstedt, F. Stability and Electronic Structure of Two-Dimensional Allotropes of Group-IV Materials. Phys. Rev. B 2015, 92, 045436.

(36) Kaur, S.; Kumar, A.; Srivastava, S.; Tankeshwar, K.; Pandey, R. Novel Phosphorus-Based 2D Allotropes with Ultra-High Mobility. Nanotechnology 2020, 31, 325702.

(37) Peng, B.; Zhang, H.; Shao, H.; Xu, Y.; Zhang, R.; Lu, H.; Zhang, D. W.; Zhu, H. FirstPrinciples Prediction of Ultralow Lattice Thermal Conductivity of Dumbbell Silicene: A 
Comparison with Low-Buckled Silicene. ACS Appl. Mater. Interfaces 2016, 8, 2097720985.

(38) Sassa, Y .; Johansson, F. O. L.; Lindblad, A.; Yazdi, M. G.; Simonov, K.; Weissenrieder, J.; Muntwiler, M.; Iyikanat, F.; Sahin, H.; Angot, T.; et al. Kagome-like Silicene: A Novel Exotic Form of Two-Dimensional Epitaxial Silicon. Appl. Surf. Sci. 2020, 530, 147195.

(39) Cahangirov, S.; Özçelik, V. O.; Xian, L.; Avila, J.; Cho, S.; Asensio, M. C.; Ciraci, S.; Rubio, A. Atomic Structure of the $\sqrt{3} \times \sqrt{ } 3$ Phase of Silicene on $\operatorname{Ag}(111)$. Phys. Rev. B 2014, 90, 035448.

(40) Curcella, A.; Bernard, R.; Borensztein, Y.; Lazzeri, M.; Prévot, G. The Mechanism for the Stabilization and Surfactant Properties of Epitaxial Silicene. Nanoscale 2018, 10, 22912300.

(41) Nečas, D.; Klapetek, P. Gwyddion: An Open-Source Software for SPM Data Analysis. Cent. Eur. J. Phys. 2012, 10, 181-188.

(42) Dawiec, A.; Garreau, Y.; Bisou, J.; Hustache, S.; Kanoute, B.; Picca, F.; Renaud, G.; Coati, A. Real-Time Control of the Beam Attenuation with XPAD Hybrid Pixel Detector. $J$. Instrum. 2016, 11, P12018.

(43) Roobol, S.; Onderwaater, W.; Drnec, J.; Felici, R.; Frenken, J. BINoculars : Data Reduction and Analysis Software for Two-Dimensional Detectors in Surface X-Ray Diffraction. $J$. Appl. Crystallogr. 2015, 48, 1324-1329.

(44) Colonna, S.; Serrano, G.; Gori, P.; Cricenti, A.; Ronci, F. Systematic STM and LEED Investigation of the Si/Ag(110) Surface. J. Phys.: Condens. Matter 2013, 25, 315301.

(45) Giannozzi, P.; Andreussi, O.; Brumme, T.; Bunau, O.; Buongiorno Nardelli, M.; Calandra, M.; Car, R.; Cavazzoni, C.; Ceresoli, D.; Cococcioni, M.; et al. Advanced Capabilities for Materials Modelling with Quantum ESPRESSO. J. Phys.: Condens. Matter 2017, 29, 465901.

(46) Prévot, G.; Hogan, C.; Leoni, T.; Bernard, R.; Moyen, E.; Masson, L. Si Nanoribbons on Ag(110) Studied by Grazing-Incidence X-Ray Diffraction, Scanning Tunneling Microscopy, and Density-Functional Theory: Evidence of a Pentamer Chain Structure. Phys. Rev. Lett. 2016, 117, 276102.

(47) Hogan, C.; Colonna, S.; Flammini, R.; Cricenti, A.; Ronci, F. Structure and Stability of Si/Ag(110) Nanoribbons. Phys. Rev. B 2015, 92, 115439.

(48) Tersoff, J.; Hamann, D. R. Theory of the Scanning Tunneling Microscope. Phys. Rev. B 1985, 31, 805-813.

(49) Leandri, C.; Le Lay, G.; Aufray, B.; Girardeaux, C.; Avila, J.; Dávila, M. E.; Asensio, M. C.; Ottaviani, C.; Cricenti, A. Self-Aligned Silicon Quantum Wires on Ag(110). Surf. Sci. 2005, 574, L9-L15.

(50) Sahaf, H.; Masson, L.; Léandri, C.; Aufray, B.; Le Lay, G.; Ronci, F. Formation of a OneDimensional Grating at the Molecular Scale by Self-Assembly of Straight Silicon Nanowires. Appl. Phys. Lett. 2007, 90, 263110.

(51) Ronci, F.; Colonna, S.; Cricenti, A.; De Padova, P.; Ottaviani, C.; Quaresima, C.; Aufray, B.; Le Lay, G. Low Temperature STM/STS Study of Silicon Nanowires Grown on the Ag(110) Surface. Phys. Status Solidi C 2010, 7, 2716-2719.

(52) Bernard, R.; Leoni, T.; Wilson, A.; Lelaidier, T.; Sahaf, H.; Moyen, E.; Assaud, L.; Santinacci, L.; Leroy, F.; Cheynis, F.; et al. Growth of Si Ultrathin Films on Silver Surfaces: Evidence of an $\operatorname{Ag}(110)$ Reconstruction Induced by Si. Phys. Rev. B 2013, 88, 121411. 
(53) Sheng, S.; Ma, R.; Wu, J.; Li, W.; Kong, L.; Cong, X.; Cao, D.; Hu, W.; Gou, J.; Luo, J.W.; et al. The Pentagonal Nature of Self-Assembled Silicon Chains and Magic Clusters on Ag(110). Nano Lett. 2018, 18, 2937-2942.

(54) Curcella, A.; Bernard, R.; Borensztein, Y.; Lazzeri, M.; Resta, A.; Garreau, Y.; Prévot, G. Multilayer Silicene: Clear Evidence of Ag-Terminated Bulk Silicon. 2D Mater. 2017, 4, 025067.

(55) Liu, Q.; Fu, Q.; Shao, X.; Ma, X.; Wu, X.; Wang, K.; Xiao, X. Diffusion of Single Au, Ag and $\mathrm{Cu}$ Atoms inside Si(111)-(7×7) Half Unit Cells: A Comparative Study. Appl. Surf. Sci. 2017, 401, 225-231.

(56) Li, S.; Wu, Y.; Tu, Y.; Wang, Y.; Jiang, T.; Liu, W.; Zhao, Y. Defects in Silicene: Vacancy Clusters, Extended Line Defects and Di-Adatoms. Sci.Rep. 2015, 5, 7881.

(57) Curcella, A.; Bernard, R.; Borensztein, Y.; Resta, A.; Lazzeri, M.; Prévot, G. Determining the Atomic Structure of the $(4 \times 4)$ Silicene Layer on $\operatorname{Ag}(111)$ by Combined GrazingIncidence x-Ray Diffraction Measurements and First-Principles Calculations. Phys. Rev. B 2016, $94,165438$.

(58) Curcella, A.; Bernard, R.; Borensztein, Y.; Resta, A.; Lazzeri, M.; Prévot, G. Structure and Stability of Silicene on $\mathrm{Ag}(111)$ Reconstructions from Grazing Incidence x-Ray Diffraction and Density Functional Theory. Phys. Rev. B 2019, 99, 205411.

(59) Feidenhans'1, R. Surface Structure Determination by X-Ray Diffraction. Surf. Sci. Rep. 1989, 10, 105-188.

(60) Bernard, R.; Borensztein, Y.; Cruguel, H.; Lazzeri, M.; Prévot, G. Growth Mechanism of Silicene on Ag (111) Determined by Scanning Tunneling Microscopy Measurements and Ab Initio Calculations. Phys. Rev. B 2015, 92, 045415.

(61) Sone, J.; Yamagami, T.; Aoki, Y.; Nakatsuji, K.; Hirayama, H. Epitaxial Growth of Silicene on Ultra-Thin Ag(111) Films. New J. Phys. 2014, 16, 095004.

(62) Satta, M.; Colonna, S.; Flammini, R.; Cricenti, A.; Ronci, F. Silicon Reactivity at the Ag(111) Surface. Phys. Rev. Lett. 2015, 115, 026102.

(63) Tsetseris, L.; Kaltsas, D. Chemical Routes to Modify, Uplift, and Detach a Silicene Layer from a Metal Substrate. Phys. Chem. Chem. Phys. 2014, 16, 5183.

(64) Du, Y.; Zhuang, J.; Wang, J.; Li, Z.; Liu, H.; Zhao, J.; Xu, X.; Feng, H.; Chen, L.; Wu, K.; et al. Quasi-Freestanding Epitaxial Silicene on $\mathrm{Ag}(111)$ by Oxygen Intercalation. Sci. Adv. 2016, 2, e1600067.

(65) Tao, L.; Cinquanta, E.; Chiappe, D.; Grazianetti, C.; Fanciulli, M.; Dubey, M.; Molle, A.; Akinwande, D. Silicene Field-Effect Transistors Operating at Room Temperature. Nat. Nanotechnol. 2015, 10, 227-231.

(66) Martella, C.; Faraone, G.; Alam, M. H.; Taneja, D.; Tao, L.; Scavia, G.; Bonera, E.; Grazianetti, C.; Akinwande, D.; Molle, A. Disassembling Silicene from Native Substrate and Transferring onto an Arbitrary Target Substrate. Adv. Funct. Mater. 2020, 30, 2004546.

(67) Xu, X.; Zhuang, J.; Du, Y.; Feng, H.; Zhang, N.; Liu, C.; Lei, T.; Wang, J.; Spencer, M.; Morishita, T.; et al. Effects of Oxygen Adsorption on the Surface State of Epitaxial Silicene on $\operatorname{Ag}(111)$. Sci. Rep. 2015, 4, 7543.

(68) Du, Y.; Zhuang, J.; Liu, H.; Xu, X.; Eilers, S.; Wu, K.; Cheng, P.; Zhao, J.; Pi, X.; See, K. W.; et al. Tuning the Band Gap in Silicene by Oxidation. ACS Nano 2014, 8, 10019-10025.

(69) Feng, J.; Liu, Y.; Wang, H.; Zhao, J.; Cai, Q.; Wang, X. Gas Adsorption on Silicene: A Theoretical Study. Comput. Mater. Sci. 2014, 87, 218-226. 
(70) Hu, W.; Xia, N.; Wu, X.; Li, Z.; Yang, J. Silicene as a Highly Sensitive Molecule Sensor for $\mathrm{NH}_{3}, \mathrm{NO}$ and $\mathrm{NO}_{2}$. Phys. Chem. Chem. Phys. 2014, 16, 6957.

(71) Prasongkit, J.; Amorim, R. G.; Chakraborty, S.; Ahuja, R.; Scheicher, R. H.; Amornkitbamrung, V. Highly Sensitive and Selective Gas Detection Based on Silicene. J. Phys. Chem. C 2015, 119, 16934-16940.

(72) Goli, M.; Ansari, R.; Rouhi, S.; Aghdasi, P.; Mozvashi, S. M. Influence of F and H Adsorption on the Elasto-Plastic Properties of Silicene: A DFT Investigation. Phys. E 2020, $119,113984$.

(73) Ding, Y.; Wang, Y. Tunable Electronic Structures of Hydrogenated Zigzag and Armchair Dumbbell Silicene Nanosheets: A Computational Study. J. Phys. Chem. C 2018, 122, 23208-23216.

(74) Zhang, T.; Zeng, Z.-Y.; Cheng, Y.; Chen, X.-R.; Cai, L.-C. Dumbbell Silicene: A StrainInduced Room Temperature Quantum Spin Hall Insulator. New J. Phys. 2016, 18, 043001. 
TOC graphic

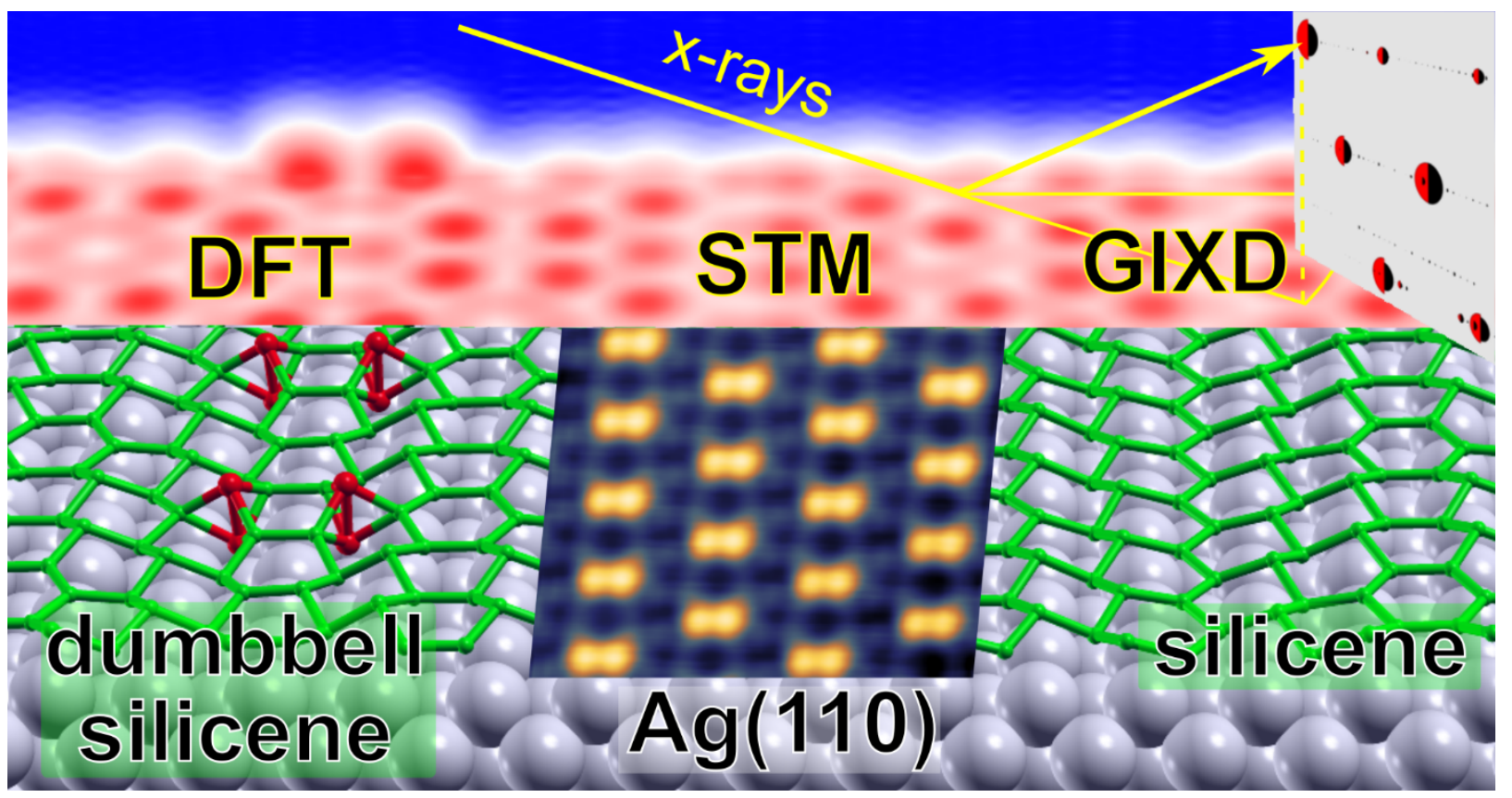

